\title{
Unit Root Testing for Bubbles: A Resurrection?
}

\author{
George A. Waters* \\ Department of Economics \\ Illinois State University \\ Normal, IL 61790-4200
}

November 3, 2007

\begin{abstract}
Evans (1991) and Charemza and Deadman (1995) present models of bubbles that are not empirically detectable by unit root tests. However, the stochastic elements of those models enter multiplicatively, while the Monte Carlo results in those papers use a linear unit root test. This paper presents results based on the more natural log specification of the simulated data and shows that bubbles generated by the stochastic explosive unit root model of Charemza and Deadman (1995) are detectable by properly specified unit root tests while those of Evans' (1991) model of periodically collapsing bubbles are not.

JEL Classification: C15, G12

Keywords: periodically collapsing bubbles, stochastic explosive root processes
\end{abstract}

*Email: gawater@ilstu.edu, Phone: 309-438-7301, Fax: 309-438-5228 


\section{Introduction}

The potential presence of rational bubbles is critical for asset pricing. Much of asset pricing theory depends on the assumption that prices are determined by fundamental information such as future dividends, but, if bubbles exist, extraneous information could have significant influence. Hence, a literature has grown around the question of the proper empirical techniques for testing for rational bubbles.

Diba and Grossman (1988) showed that linear unit root tests can empirically detect a simple form of rational speculative bubbles. Evans (1991) and Charemza and Deadman (1995) develop more general models of rational bubbles that resist such detection. However, in both models, the stochastic terms enter multiplicatively, while the econometric model behind the test is a linear random walk. The present work examines whether unit root tests on the more natural log specification of the data simulated from the model of Evans (1991) and Charemza and Deadman (1995) can identify the presence of bubbles. The central result of the paper is that bubbles generated by the stochastic explosive unit root model of Charemza and Deadman (1995) can be detected by properly specified unit root tests, but the periodically collapsing bubbles of Evans (1991) cannot.

The standard model for asset price $p_{t}$ with dividend $d_{t}$ assumes

$$
p_{t}=(1+r)^{-1} E_{t}\left(p_{t+1}+d_{t+1}\right)
$$

for a constant discount rate $(1+r)^{-1}$. Under rational expectations, the solution for the price can be written

$$
p_{t}=\sum_{j=1}^{\infty}(1+r)^{-j} E_{t} d_{t+j}+b_{t}
$$

where $b_{t}$ is the bubble term. The strong efficient markets hypothesis assumes that $b_{t}=0$ so that the price is determined solely by the present value of expected future dividends. However, rational expectations admits any $b_{t}$ that satisfies the sub-martingale condition $E_{t-1}\left(b_{t}\right)=(1+r) b_{t-1}$.

Under the further assumption that $b_{t}$ follows a linear ARMA process, the case examined in Diba and Grossman (1988), the bubble diverges and exceeds any given threshold given enough time. However, other more plausible, though more complicated, models for $b_{t}$ are available, those of Charemza and Deadman (1995) and Evans (1991) being prominent examples. We examine each in turn. 


\section{Stochastic Explosive Unit Root Processes}

The model proposed by Charemza and Deadman (1995) for a bubble process $b_{t}$ is as follows.

$$
b_{t}=\theta_{t} b_{t-1} u_{t}
$$

The random variables $\theta_{t}$ and $u_{t}$ have means $1+r$ and 1 , respectively, so the sub-martingale property $E_{t-1}\left(b_{t}\right)=(1+r) b_{t-1}$ is satisfied. To ensure non-negativity, they are assumed to have the form $\theta_{t}=$ $\exp \left(\Theta_{t}\right)$ and $u_{t}=\exp \left(U_{t}\right)$, where $\Theta_{t} \sim i i d\left(\ln (1+r)-\frac{1}{2} \sigma_{\Theta}^{2}, \sigma_{\Theta}^{2}\right)$ and $U_{t} \sim i i d\left(-\frac{1}{2} \sigma_{U}^{2}, \sigma_{U}^{2}\right)$.

This stochastic explosive root model (1) (STER) can alternate between explosive and stationary behavior for realizations of $\theta_{t}$ above or below one. Since $\theta_{t}$ is stochastic, there may be temporary bursts of explosive behavior. Hence, it is a parsimonious model of a non-divergent bubble without requiring an exogenous probability of collapse.

As Charemza and Deadman (1995) point out, for a constant $\theta_{t}$, the model (1) in logarithms is equivalent to a standard linear random walk with drift. The associated econometric model can be written as

$$
\widetilde{b}_{t}=\mu+\rho \widetilde{b}_{t-1}+\varepsilon_{t}
$$

The model of a bubble (1) is equivalent to (2) for $\widetilde{b}_{t}=\ln b_{t}, \mu=\theta_{t}=1+r, U_{t}=\varepsilon_{t}$ and $\rho=1$. Following Evans (1991), Charemza and Deadman (1995) simulate (1) for various parameter values and test for $\rho=1$ in (2) and conclude that the various unit root test cannot detect bubbles of this form. However, they conduct these test on the levels of $b_{t}$ directly, even though the linear model is closely related to the log of $b_{t}$.

The present work reports results of tests for unit roots on the log of $b_{t}$ from simulations of the STER model (1). With the relatively small sample of 100 periods and the inclusion of drift, since $r$ could be positive, the most appropriate test uses the $N_{2}$ statistic of Bhargava (1986). For comparison purposes, we also use a $t$ test, comparing with the critical values from Dickey-Fuller (1976). Both are two-sided tests of the null $\rho=1$ in (2), and we report results for both alternatives $\rho<1$ and $\rho>1$. Note that the interpretation of the tests is somewhat different with the log specification. For the tests on levels in Charemza and Deadman (1995), Diba and Grossman (1986), and Evans (1991), rejection in favor of $\rho>1$ is evidence of a bubble, while in logs, failure to reject $\rho=1$ is evidence of a bubble ${ }^{1}$. The primary issue remains whether the tests mistakenly reject in favor of the stationary alternative $\rho<1$.

One difficulty in simulating the STER model (1) is that $b=0$ is an absorbing value, though the model is designed to fluctuate around one during non-explosive times. In many sample simulations, once $b_{t}$ enters

\footnotetext{
${ }^{1}$ The term 'explosive' is avoided, since both cases where $\rho=1$ and $\rho>1$ could be considered explosive in the log specification.
} 
the neighborhood of zero, it rarely leaves. The vast majority of these simulations are stationary according to the econometric tests for unit roots and could easily bias the results, particularly for higher variances of the stochastic terms. Therefore, we throw out the simulations where $b_{t}$ is less than 0.01 for the last 10 periods.

As in Charemza and Deadman (1995), we compute critical values, shown in Table 1 below, that give a significance level of $5 \%$ for varying values ${ }^{2}$ of $\sigma_{\Theta}^{2}$, fixing $\sigma_{U}^{2}$ at $\sigma_{U}^{2}=0.02$. These can be compared with the standard values for the linear random walk model of -3.45 for the Dickey-Fuller test and 0.26 for the $N_{2}$ statistic from Bhargava (1986) in the case of the stationary alternative $\rho<1$. The prob columns for both alternatives report the fraction of rejections of the null $\rho=1$ using the standard critical values $N_{2}=0.26$ and $N_{2}=0.022$, respectively. Each row was computed using 25,000 trials. The skips column records how may trials were thrown out before achieving 25,000 to use.

As expected, the simulated critical values for $r=0$ and $\sigma_{\Theta}^{2}=0$ in the first row, which corresponds to the linear random walk model (2), are quite close to the standard values. Furthermore, the prob columns is close to the specified $5 \%$ level for the linear random walk case.

\begin{tabular}{|c|c|c|c|c|c|c|c|c|}
\hline \multirow[b]{2}{*}{$r$} & \multirow[b]{2}{*}{$\sigma_{\theta}$} & \multirow[b]{2}{*}{ skips } & \multicolumn{3}{|c|}{ Alternative: $\rho<1$} & \multicolumn{3}{|c|}{ Alternative: $\rho>1$} \\
\hline & & & DF & $\mathrm{N}_{2}$ & prob & DF & $\mathrm{N}_{2}$ & prob \\
\hline 0 & 0 & 0 & -3.444 & 0.266 & 0.050 & -0.905 & 0.022 & 0.051 \\
\hline 0.03 & 0.1 & 0 & -3.453 & 0.267 & 0.050 & -0.923 & 0.022 & 0.049 \\
\hline 0.03 & 0.15 & 0 & -3.458 & 0.264 & 0.049 & -0.906 & 0.022 & 0.049 \\
\hline 0.03 & 0.2 & 46 & -3.453 & 0.263 & 0.048 & -0.922 & 0.022 & 0.052 \\
\hline 0.03 & 0.25 & 790 & -3.458 & 0.265 & 0.049 & -0.896 & 0.022 & 0.051 \\
\hline 0.03 & 0.3 & 3605 & -3.452 & 0.264 & 0.049 & -0.932 & 0.022 & 0.048 \\
\hline 0.03 & 0.4 & 24,255 & -3.431 & 0.261 & 0.046 & -0.895 & 0.022 & 0.052 \\
\hline 0.03 & 0.5 & 100,763 & -3.440 & 0.262 & 0.047 & -0.890 & 0.022 & 0.052 \\
\hline
\end{tabular}

Table 1

The surprising result is the computed critical values and rejection probabilities are similar for $r=0.03$ and higher values of $\sigma_{\Theta}^{2}$, in contrast to the results in Charemza and Deadman (1995), who find critical values both higher and lower than the standard values and rejection probabilities quite different from $5 \%$. Therefore, using the log specification and accounting for drift, unit root testing does not reject the null with high probability, giving evidence of bubbles for data generated by the STER model (1).

\footnotetext{
${ }^{2}$ For the simulations, the the stochastic term $\Theta_{t}$ and $U_{t}$ are normally distributed.
} 
This conclusion is robust for the alternative $\rho>1$ as well. The computed critical values match the standard ones and the rejection probabilities are all close to $5 \%$. One might suspect that these results are driven by the elimination of trials where $b_{t}$ approaches zero. However, the cases where $\sigma_{\Theta}^{2}=0.15$ and 0.2 do not deviate significantly and the number of skips is zero or negligible. The large number of skips in the cases with high values of $\sigma_{\Theta}^{2}$ does suggest that $b_{t}$ collapsing toward zero for some trials may be the source of some of the extreme computed critical values in Charemza and Deadman (1995).

\section{Periodically Collapsing Bubbles}

Since bubbles generated by the STER model (1) are detectable by unit root tests under the log specification, the obvious next question is whether periodically collapsing bubbles of Evans (1991) have the same property. Although the stochastic terms enter multiplicatively in the model of periodically collapsing bubbles, as in the STER model (1), the following simulation results indicate that periodically collapsing bubbles are not detectable by such an approach.

In the model of periodically collapsing bubbles (PCB), the bubble variable $b_{t}$ evolves according to the following two equations.

$$
\begin{aligned}
& b_{t}=(1+r) b_{t-1} u_{t} \quad \text { if } b_{t} \leq \alpha \\
& b_{t}=\left[\delta+\pi^{-1}(1+r) \psi_{t}\left(b_{t-1}-(1+r)^{-1} \delta\right)\right] u_{t} \quad \text { if } b_{t}>\alpha
\end{aligned}
$$

The parameter $r$ and the stochastic term $u_{t}$ are the same as in the STER model (1). The stochastic term $\psi_{t}$ has binomial distribution such that it equals 1 with probability $\pi$ and 0 with probability $1-\pi$. The parameters $\delta$ and $\alpha$ are both positive and satisfy the condition $\delta<(1+r) \alpha$.

The PCB process (3) can switch between two regimes depending on the threshold parameter $\alpha$. As long as $b_{t}$ remains below $\alpha$, it grows at mean rate $1+r$ but if $b_{t}$ rises above $\alpha$ it grows at the faster mean rate $(1+r) \pi^{-1}$ as long as $\psi_{t}$ is 1 . When $\psi_{t}$ is 0 , the bubble collapses and falls to $\delta$ in expectation. The sub-martingale property $E_{t-1}\left(b_{t}\right)=(1+r) b_{t-1}$ is again satisfied, though $b_{t}$ could grow at a rate faster than $1+r$ for some periods of time. The duration and the rate of growth of the fast growth regime depend on the probability of collapse $\pi$.

As with the STER model, the PCB model (3) is closely related to the linear random walk with drift. If $\pi$ is fixed at 1 , both regimes are equivalent to the econometric random walk model (2) for $\widetilde{b}_{t}=\ln b_{t}, \mu=1+r$, $U_{t}=\varepsilon_{t}$ and $\rho=1$. Again, we create simulated data for $b_{t}$ using (3) and test for $\rho=1$ in (2) on the $\log$ of $b_{t}$. The central question is whether the switching between the two regimes for $\pi<1$ impacts the ability of 
the tests to detect bubbles.

Table 2 reports results for varying $r$ and $\pi$. As in Table 1, the first row with $r=0$ closely approximates the linear random walk model (2) since $\pi$ is very close to one so both regimes are nearly identical, and the computed critical values are very close to the standard critical values for the Dickey-Fuller (1976) test and the $N_{2}$ statistic (Bharghava 1986), and the rejection probabilities using the standard critical values for $N_{2}$ are near $5 \%$.

\begin{tabular}{rr|rrr|rrr|} 
& & \multicolumn{3}{|c|}{ Alternative: $\rho<1$} & \multicolumn{3}{c|}{ Alternative: $\rho>1$} \\
\hline \hline 0 & 0.999 & -3.445 & 0.263 & 0.048 & -0.928 & 0.022 & 0.047 \\
0.05 & 0.999 & -3.650 & 0.144 & 0.005 & -0.014 & 0.016 & 0.136 \\
0.05 & 0.99 & -3.097 & 0.170 & 0.008 & 0.342 & 0.013 & 0.158 \\
0.05 & 0.95 & -3.467 & 0.261 & 0.044 & -0.923 & 0.029 & 0.040 \\
0.05 & 0.85 & -4.338 & 0.426 & 0.427 & -1.902 & 0.086 & 0.006 \\
0.05 & 0.75 & -4.908 & 0.540 & 0.800 & -2.707 & 0.171 & 0.006 \\
0.05 & 0.5 & -5.893 & 0.756 & 0.958 & -3.935 & 0.282 & 0.001 \\
0.05 & 0.25 & -6.746 & 0.959 & 0.957 & -4.261 & 0.280 & 0.000
\end{tabular}

Table 2

For $r=0.05$, as in Evans (1991), the critical values and rejection probabilities are not standard and vary with $\pi$. For $\pi=0.999$ the $N_{2}$ statistic is able to reject in favor of the stationary alternative $\rho<1$ almost every time, as in Evans (1991), showing evidence of a bubble. In a sense, this test works too well, since the rejection probability is below the specified $5 \%$ significance level. This case is similar to the results in Diba and Grossman (1988) as well, though we interpret failure to reject either alternative as evidence of a bubble, since we test the log of $b_{t}$, while they interpret only rejection in favor of their explosive alternative $\rho>1$ (testing in levels) as evidence of a bubble ${ }^{3}$.

For lower values of $\pi$, meaning there are more frequent collapses, the test is less able to reject in favor or the stationary alternative $\rho<1$, shown by the increasing rejection probabilities and magnitudes of the computed critical values. The results are qualitatively similar to Evans (1991), though the change in the probabilities is less dramatic. For example, for $\pi=0.85$, using the $N_{2}$ statistic on the level of $b_{t}$, Evans

\footnotetext{
${ }^{3}$ For the test of the $\log$ of $b_{t}$, rejection in favor of the alternative $\rho>1$ may be interpreted as evidence of a bubble though possibly generated by a process other than the PCB model. This is an subtle and interesting issue, but does not affect the primary arguments of the paper.
} 
finds rejection in favor or the stationary alternative in $86.5 \%$ of the trials, while our test on the $\log$ of $b_{t}$, rejects in only $42.7 \%$, which is still a serious failure of the test to detect bubbles. For $\pi \geq 0.95$, the test on the $\log$ of $b_{t}$ performs well, but there is no reason to believe that lower values of $\pi$ are less relevant.

\section{Conclusion}

While appropriately specified unit root tests on the log of $b_{t}$ are able to detect bubbles of the form of the STER model (1), bubbles generated by the PCB model (3) are not generally detectable. As Evans (1991) states, unit root tests have difficulty differentiating between periodically collapsing bubbles and a persistent, mean-reverting process.

For empirical researchers, the issue of testing for bubbles remains clouded. Evans' (1991) model has spawned a number of econometric tests designed to detect periodically collapsing bubbles in the data, such as the momentum threshold autoregressive approach of Bohl (2003) and the residuals augmented Dicky-Fuller test of Taylor and Peel (1998). However, the PCB model is quite restrictive and whether these tests could detect bubbles of other forms is an open question. Furthermore, these tests can yield conflicting results in applied work as in Payne and Waters (2005a,b).

One could take the position that the PCB model is too stylized to be relevant for data analysis and use unit root tests in spite of Evans' (1991) arguments. For researchers of this opinion, the present work offers a caveat about proper specification of such tests. Ultimately, the issue of testing for bubbles should be analyzed for other models of bubbles, such as the model in Parke and Waters (2005), with deeper theoretical foundations beyond the econometric specifications in this paper.

\section{References}

Bohl, M.T., 2003, Periodically collapsing bubbles in the U.S. stock market? International Review of Economics and Finance 12, 385-397.

Bhargava, A., 1986, On the theory of testing for unit roots in observed time series, Review of Economic Studies 53, 369-84.

Charemaza, W. and D. Deadman, 1991, Speculative bubbles with stochastic explosive roots: The failure of unit root testing, Journal of Empirical Finance 2, 153-163.

Diba. T, and H. Grossman,1988, Explosive rational bubbles in stock prices?, The American Economic Review $78,520-530$.

Evans G. Pitfalls in testing for explosive bubbles in asset prices. The American Economic Review 1991;81;92230

Fuller, W. A., 1976, Introduction to Statistical Time Series. (Wiley, New York). 
Parke, W. and G. Waters, 2007, An Evolutionary game theory explanation of ARCH, Journal of Economic Dynamics and Control 31(7), 2234-2262.

Payne, J. and G. Waters, 2005a, Have equity REITs experiences periodically collapsing bubbles? Journal of Real Estate Finance and Economics 34(2), 207-224.

Payne, J. and G. Waters, 2005b, REIT markets and rational speculative bubbles: an empirical investigation, Applied Financial Economics, forthcoming.

Taylor, M. and D. Peel, 1998, Periodically collapsing stock price bubbles: A robust test, Economics Letters 61, 221-228. 